\title{
Mail-in and remote access for solution scattering at the LiX beamline
}

\author{
L Yang ${ }^{1}$, S Chodankar ${ }^{1}$, J Byrnes ${ }^{1}$ \\ ${ }^{1}$ Brookhaven National Laboratory, Upton, NY \\ lyang@bnl.gov
}

At the Life Science X-ray Scattering (LiX) beamline at NSLS-II, we have developed capabilities for fully automated solution scattering data collection and data processing [1]. While it has been our plan all along to support mail-in and remote user access, the COVID-19 pandemic has made it a necessity. We report our current status in preparation for supporting remote user experiments, which we will make available to users for the 2020-2 cycle starting in mid-July. Central to our implementation is an Opentron liquid handling robot that we use to transfer samples from standard 96-well plates to our 18-position sample holder. Sample plates and sample holders are tracked using bar codes and QR codes, respectively, to ensure accurate identification of the samples. Remote access is realized through NX. Once the users' samples are ready for measurements, the user is allowed to run a simple GUI to start measurements and monitor the process of data collection. Alternatively, overnight static measurements can be started by beamline staff on all samples that have been accumulated. Additional software tools have been developed to ensure data quality and assist data analysis. As soon as the processed data in hdf5 file and analysis results are available, they can be accessed either from the users' home institution via Globus, or using the computing resources at NSLS-II. [1] Solution scattering at the Life Science X-ray Scattering (LiX) beamline L. Yang, S. Antonelli, S. Chodankar, J. Byrnes, E. Lazo and K. Qian J. Synchrotron Rad. (2020). 27, 804-812, https://doi.org/10.1107/S1600577520002362

Acta Cryst. (2020). A76, a163 